\title{
Determining the Dimension of Subsurface Defect by Active IR - Experimental Research
}

\author{
S. Gryś \\ Czestochowa University of Technology, Faculty of Electrical Engineering, \\ Al. Armii Krajowej 17, PL 42-200 Częstochowa, Poland, e-mail: grys@el.pcz.czest.pl
}

\begin{abstract}
:
This paper presents research on method of processing thermal images for detecting and characterizing material defects or nonuniformities in the internal structure of material. An active thermography was chosen as a NDT method. The hidden defects were revealed by analysis of temperature field of the front surface of tested material that was externally excited with heating lamps. The background removal and image segmentation were applied to the last thermogram from the sequence of thermogram recorded at the end of the heating phase. The paper focuses on accuracy of determination of transversal dimension of the subsurface flaws in Plexiglas slab with drilled bottom holes imitating the defects. The following factors influencing accuracy are taken into account: defect depth, the emissivity of the surface inspected as input parameter being set by user to the IR camera, the type of filtering used to remove an effect of the non-uniformity of heating the object surface with incandescent lamp.
\end{abstract}

Key words: nondestructive testing, active thermography, defect characterization, automatic image processing, segmentation.

\section{Introduction}

Nowadays, it is increasing the use of materials such as composites and multilayered or honeycomb structures, due to their good physical properties in relation to the low weight. Their use in very demanding industry branches as aerospace, naval or automotive imposes the necessity of rigorous examination all parts during production phase and exploitation. The nondestructive testing is suggested in the cases when there is no permission to stop the production line or object under examination must remain in service with no changed properties. The first level of testing routine is visual control performed by operator or specialized vision systems working in the visual range. Later, objects are checked for hidden structural defects using some commonly accepted NDT techniques, e.g. radiographic, ultrasound methods, active thermography and others.

With respect to the location of the defect relative to the object surface, material defects can be divided into surface, subsurface, internal and with respect to the shape flat or volumetric. The distinction into discontinuities originated during production and exploitation is commonly used. These involve, respectively tearing the macrostructure and possibly the microstructure of the object due to a faulty production process, object fatigue in the course of normal exploitation. To the first category belong structural damages, or cavities caused by mechanical or chemical factors, or internal stress. Exploitation damages, on the other hand, can occur due to static, dynamic and shock loads caused by external forces, corrosion, erosion, thermal shock, chemical reactions or mechanical friction. Considering the consequences for exploitation, the most dangerous non-uniformities are flat, parallel to the object surface. The closer they are to the surface, the more serious the defect is, since the majority of critical stresses causing cracks occur on the surface, or immediately under it. Solid metallic and non-metallic intrusions of acute shape, overlaps and delaminations are also dangerous. Other material defects include geometrical discontinuities involving change of thickness, shape, or cavities originating from production, or from fatigue.

In order to carry out a non-destructive test effectively it is necessary to find a diagnostic method appropriate for the kind of material inspected. Determination of selected parameters of flaws detected is essential for recognizing reasons of their occurrence and risk assessment of object destruction during further operation. These defect parameters include the depth at which they are located, 
their geometrical dimensions and their thermal properties as compared to those of the material inspected [1-4].

The results of 3D simulation of externally heated sample and way how to detect the boundary of defects was presented in [5]. This paper focuses of determination of transversal dimension (size) of detected material flaws. The theoretical results are verified for real measurements carried out for Plexiglas slab Fig. 1. From the bottom side of the sample the 9 holes were drilled to emulate the occurrence of defects. They have the same shape of a hollow cylinder with $10 \mathrm{~mm}$ diameter and are located at various depth. The thermographic system was used to observe a front surface being excited with external heaters. To ensure the good conditions for IR inspection, the inspected surface covered with a black mat paint of high emissivity.

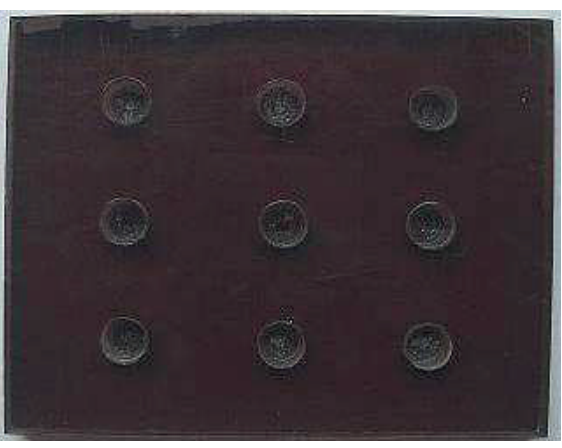

Fig.1. Slab of Plexiglas; view from the bottom side with visible 9 holes imitating flaws (mirrored horizontally).

\section{Data processing path}

To detect the flaws and assess their parameter some new techniques were invented and

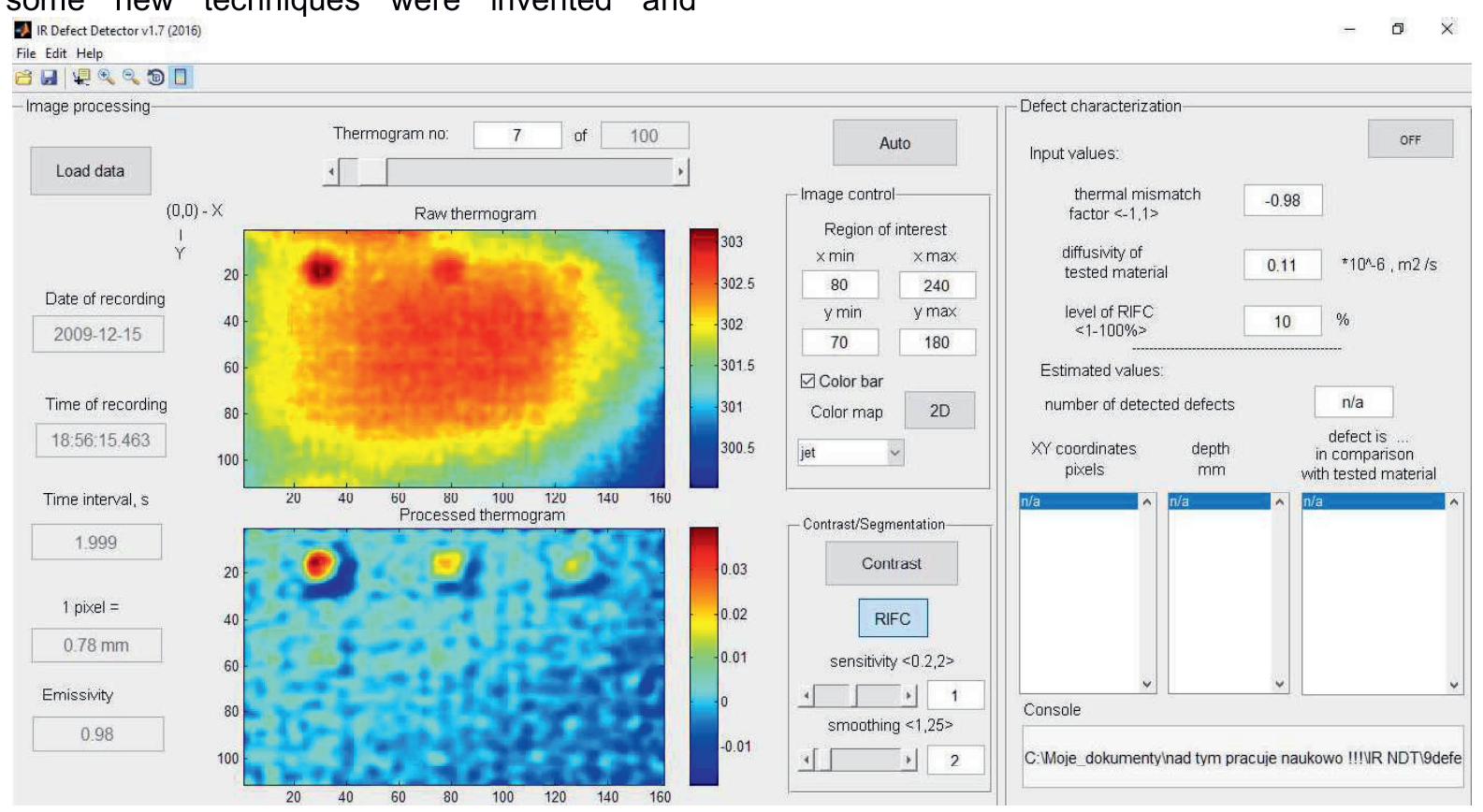

Fig. 3. View of the IR Defect Detector with inactive option "Defect characterization" for early phase of heating. combined with others commonly known in the field of signal processing. They are: region of interest, digital filtering, automatic object counting and reporting, fitting experimental data to 1D analytical model of physical behaviour. The simplified version of data processing path is illustrated in Fig. 2 with bolded elements related to the task, i.e. determination of transversal size of defect. More details of data processing path and algorithms were given in [6].

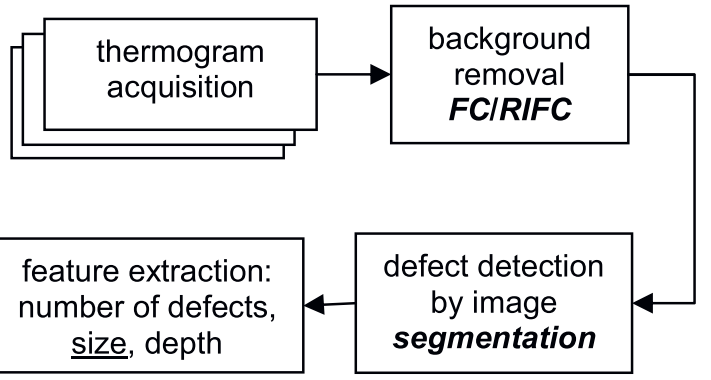

Fig. 2. Processing path (simplified)

To ease the processing of sequence of collected thermograms a dedicated software, that was named "IR Defect Detector", was created. This software tool was built in MatLab environment. In Fig. 3 the GUI is shown for the case of disabled option of automatic "Defect characterization". Some defects stay better visible at lower image after non-uniform background estimation and removal as a result of operation of FC or RIFC contrast enhancement techniques. These formulas are used for noise suppression and to remove an effect of the non-uniformity of heating the object surface with incandescent lamp. 
It can be caused by not constant density of absorbed energy or emissivity of tested material. The filtered contrast $F C$ technique $[6,7]$ is based on classical un-sharp filtering as eq. (1).

$$
F C=T-\operatorname{filter}(T)
$$

Every pixel with $(x, y)$ coordinates of the thermogram recorded at the time $t$ is processed with above formula. The role of the filtering is to remove the influence of the defect (seen as hot or cold object) on the temperature distribution on the object surface, by approximating the background as in a defect-free thermogram. A optimal filter should mask the presence of a defect. Additionally, a filter should have a noisereducing ability. The smoothed version of the image is typically obtained by filtering the original one with a mean, median or a Gaussian filter kernel. The morphological operation i.e. erosion followed by dilation with the same structuring element can be also applied. In this work the two-dimensional Gaussian kernel was employed. Its notation in the vector form is given by eq. (2).

$$
f(\mathbf{i}, \mathbf{B})=\exp \left(-\frac{1}{2} \cdot(\mathbf{i}-\boldsymbol{\mu})^{T} \operatorname{cov}^{-1}(\mathbf{i}-\boldsymbol{\mu})\right)
$$

where i states the vector of the independent variables, and $\boldsymbol{\mu}$ is the vector of the central values, B - the width vector, cov - the covariance matrix. The function parameters are described by eq. (3), where $r_{i j}$ is the correlation measure of the variables $i, j$ determinated in the interval $i=<1 \div i_{\max }>$ and, respectively, $j=<1 \div j_{\max }>$. It was assumed $i_{\max }=7 \cdot B_{i}+1, j_{\max }=7 \cdot B_{j}+1$ and for simplicity: $i_{\text {max }}=j_{\text {max }}$ and $B=B_{i}=B_{j}$.

$$
\begin{aligned}
& \mathbf{i}=\left[\begin{array}{l}
i \\
j
\end{array}\right] \quad \boldsymbol{\mu}=\frac{1}{2}\left[\begin{array}{c}
i_{\text {max }} \\
j_{\max }
\end{array}\right] \quad \mathbf{B}=\left[\begin{array}{l}
B_{i} \\
B_{j}
\end{array}\right] \\
& \operatorname{cov}=\left[\begin{array}{cc}
\mathbf{B}_{i}^{2} & r_{i j} \cdot \mathbf{B}_{i} \cdot \mathbf{B}_{j} \\
r_{i j} \cdot \mathbf{B}_{i} \cdot \mathbf{B}_{j} & \mathbf{B}_{j}^{2}
\end{array}\right]
\end{aligned}
$$

It is necessary to norm the filter coefficients to assure filter gain is one as shown in eq. (4) and $i, j$ and $B$ are scalars now.

$$
f_{\text {scal }}(i, j, B)=\frac{f(i, j, B)}{\sum_{i=1}^{i_{\max }} \sum_{j=1}^{j_{\max }} f(i, j, B)}
$$

The filtering operation filter $(T)$ is performed by a 2-D discrete convolution of the function $f_{\text {scal }}(\mathbf{i}, \mathbf{B})$ and temperature read from thermogram. An alternative to $2 \mathrm{D}$ filtering is double $1 \mathrm{D}$ filtering. The rows of the image are first filtered with the
1D filter, and the resulting filtered image is then filtered column-wise by the 1D column filter.

An another formula of contrast, that was proposed and discussed previously by author [6] is relative incremental filtered contrast RIFC that is expressed by eq. (5).

$$
R I F C=\frac{T-\text { filter }(T)}{\text { filter }(T)-T_{\text {ref }}}
$$

The temperature $T_{\text {ref }}$ is calculated as an arithmetic mean from the reference thermogram, typically from before the heating phase.

Next phase of image processing path, similar to that presented in [8], is segmentation technique. Assuming the existence of two classes only, the segmentation is identical to binarization of the image. Each pixel is classified either as background, i.e. defect free area, or as defect, expressed symbolically as the colour white or black. The effect of segmentation applied to thermogram of tested sample was illustrated in Fig. 4. The defect no. 9 is deeply located than defect no. 8 , etc., and the defect no. 1 is the shallowest one. The global thresholding was applied with Otsu method for evaluating its optimal level.

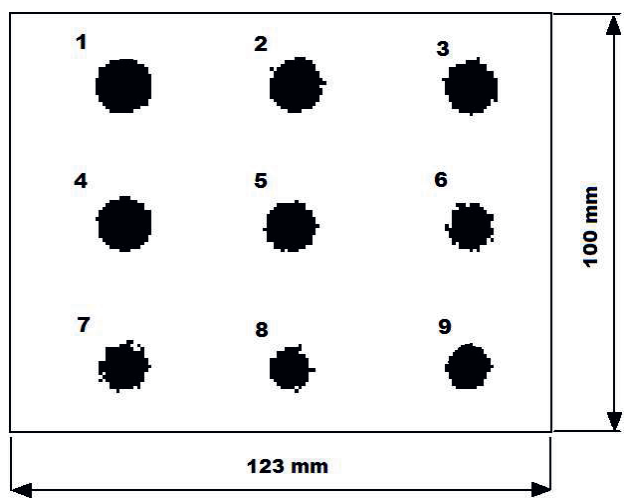

Fig. 4. Effect of segmentation for all 9 defects.

\section{Results and discussion}

In this chapter an attempt is made to assess the accuracy of determining the diameter of the each defect. The following factors are taken into account: defect depth, the emissivity $\varepsilon$ of the surface inspected as input parameter being set by user to the IR camera, the value of filter $B$ coefficient and the type of filtered contrast applied to process image FC or RIFC. For assumed experimental condition the pixel granularity of thermal image is $0.78 \mathrm{~mm} / \mathrm{pixel}$ and finally the defect diameter corresponds to the 12,8 pixels $\approx 13$ pixels. Table 1 presents the results of analysis of a single thermogram recorded at the end of the heating phase, i.e. at $t=120 \mathrm{~s}$. This time is fully enough to reveal all the defects [9]. The shadowed values belong to 
the $\pm 20 \%$ of the "true value" and are in range of $[11 \div 15]$ pixels. The values differing by not more than 1 pixel, i.e. $[12,13,14]$ are in bold type.

The conclusions from the experiment conducted for tested sample are as follows:

- The accuracy of defect size determination only slightly depends on the emissivity value set by IR camera operator.

- The choice of filtered contrast FC or RIFC almost does not affect the accuracy of defect size determination.

- The accuracy of defect size determination does not significantly depend on the value of $B$ in the assumed range of its variation $(B=15$ corresponds to the defect width in pixels for tested sample of material).

- Defects located deeper under the surface were correctly characterised with higher values of the parameter $B$. By selecting $B$ individually for each defect it is possible to compensate the effect of underestimation or overestimation of the transverse dimension, which demonstrates that the value of the parameter does affect the accuracy of the method to some extent.

Tab. 1: Evaluated horizontal dimension of defects from segmented thermogram, pixels

a) emissivity $\varepsilon=0,98$ (close to real value)

\begin{tabular}{|c|c|c|c|c|c|c|c|c|c|c|}
\hline \multirow{2}{*}{\multicolumn{2}{|c|}{$t=120 \mathrm{sec}$}} & \multicolumn{9}{|c|}{ Defect no. } \\
\hline & & 1 & 2 & 3 & 4 & 5 & 6 & 7 & 8 & 9 \\
\hline$F C$ & \multirow{2}{*}{$B=5$} & 14 & 13 & 13 & 13 & 12 & 11 & 10 & 9 & 10 \\
\hline RIFC & & 14 & 14 & 14 & 13 & 12 & 11 & 10 & 9 & 10 \\
\hline$F C$ & \multirow{2}{*}{$B=10$} & 16 & 15 & 14 & 16 & 14 & 11 & 12 & 9 & 10 \\
\hline RIFC & & 16 & 16 & 15 & 16 & 14 & 11 & 11 & 9 & 10 \\
\hline$F C$ & \multirow{2}{*}{$B=15$} & 17 & 18 & 16 & 16 & 16 & 13 & 13 & 12 & 12 \\
\hline RIFC & & 17 & 18 & 16 & 16 & 16 & 13 & 13 & 12 & 12 \\
\hline$F C$ & \multirow{2}{*}{$B=20$} & 17 & 16 & 17 & 17 & 16 & 13 & 14 & 12 & 11 \\
\hline RIFC & & 17 & 16 & 17 & 17 & 16 & 13 & 14 & 12 & 12 \\
\hline
\end{tabular}

b) emissivity $\varepsilon=0,50$ (far to real value)

\begin{tabular}{|c|c|c|c|c|c|c|c|c|c|c|}
\hline \multirow{2}{*}{\multicolumn{2}{|c|}{$t=120 \mathrm{sec}$}} & \multicolumn{9}{|c|}{ Defect no. } \\
\hline & & 1 & 2 & 3 & 4 & 5 & 6 & 7 & 8 & 9 \\
\hline$F C$ & \multirow{2}{*}{$B=5$} & 13 & 14 & 13 & 13 & 14 & 12 & 10 & 9 & 9 \\
\hline RIFC & & 13 & 14 & 13 & 13 & 14 & 12 & 10 & 9 & 9 \\
\hline$F C$ & \multirow{2}{*}{$B=10$} & 16 & 15 & 18 & 14 & 14 & 11 & 11 & 9 & 10 \\
\hline RIFC & & 16 & 15 & 18 & 14 & 14 & 11 & 11 & 9 & 10 \\
\hline$F C$ & \multirow{2}{*}{$B=15$} & 17 & 17 & 16 & 18 & 16 & 13 & 16 & 12 & 12 \\
\hline RIFC & & 17 & 17 & 16 & 18 & 16 & 13 & 16 & 12 & 12 \\
\hline$F C$ & \multirow{2}{*}{$B=20$} & 18 & 17 & 16 & 17 & 17 & 13 & 13 & 12 & 11 \\
\hline RIFC & & 18 & 17 & 17 & 17 & 17 & 13 & 13 & 11 & 11 \\
\hline
\end{tabular}

\section{Conclusion}

From practical point of view the main conclusions are the proposed method is robust to incorrect estimation of material emissivity as input parameter of IR camera and robust to unmatching the filter parameter $B$ to the defect dimension. $F C$ formulae, as a simpler and faster technique, should be used instead of RIFC if defect depth is not estimated as another required flaw parameter. The next scheduled research in the near future is testing many real defective materials to speed up the process of its validation as robust image processing technique being a part of active TNDT method.

\section{References}

[1] S. Bagavathiappan and et. al,Infrared thermography for condition monitoring - A review, Infrared Physics \& Technology 60 (2013), 35-55; doi: 10.1016/j.infrared.2013.03.006

[2] B. Milovanovic, I. Banjad Pecur, Review of Active IR Thermography for Detection and Characterization of Defects in Reinforced Concrete, J. Imaging 2016, 2, 11; doi: 10.3390/jimaging2020011

[3] C. Ibarra-Castanedo, Comparative Study of Active Thermography Techniques for the Nondestructive Evaluation of Honeycomb Structures, Research in Nondestructive Evaluation 20, (2009), Issue 1,; doi: $10.1080 / 09349840802366617$

[4] H. Wiggenhauser, Active IR-applications in Civil Engineering, Infrared Physics \& Technology 43, Issues 3-5, 233-238 (2002); doi: 10.1016/S13504495(02)00145-7

[5] S. Gryś, L. Vokorokos, L. Borowik, Size Determination of Subsurface Defect by Active Thermography - Simulation Research, Infrared Physics \& Technology 62, 147-153 (2014); doi: 10.1016/j.infrared.2013.11.011

[6] S. Gryś, Filtered thermal contrast based technique for testing of material by infrared thermography, Opto-Electronics Review 19 (2), 234-241 (2011); doi: 10.2478/s11772-011-0009-3

[7] S. Gryś, New Thermal Contrast Definition for Defect Characterisation by Active Thermography, Measurement 45, 1885-1892 (2012); doi: 10.1016/j.measurement.2012.03.017

[8] P. Venegas and et. al, Image and Data Processing Techniques Applied to Infrared Thermographic Non-Destructive Inspections of Aeronautical Composite Components, 4th Int. Symp. on NDT in Aerospace 2012 - We.2.A.1.

[9] S. Gryś, W. Minkina, L. Vokorokos, Automated Characterisation of Subsurface Defects by Active IR Thermographic Testing - Discussion of Step Heating Duration and Defect Depth Determination, Infrared Physics \& Technology 68, 84-91 (2015); doi: 10.1016/j.infrared.2014.11.005 